\title{
Investigation and Analysis of Nursing Intern's Willingness to Practice in Pediatrics
}

\author{
Kokula Krishna Hari Kunasekaran ${ }^{1}$ and Wang $\operatorname{Min}^{2}$ \\ ${ }^{1}$ Association of Scientists, Developers, and Faculties (ASDF), United Kingdom \\ ${ }^{2}$ Department of Nursing, Mudanjiang Medical University, China \\ 1prm@kokulakrishnaharik.in, ${ }^{2}$ wangminmyy1966@163.com
}

\begin{abstract}
In pediatrics, nursing interns are an important reserve force for nursing work. The willingness to practice in pediatrics is important to nursing effect and future development for pediatrics. This study selected 216 pediatric nursing interns as the study samples and investigate their pediatric nursing cognition and pediatric nursing willingness. The survey confirmed that $\geq 60 \%$ of the interns agreed that pediatric nursing has the following Features: pediatric nursing requires high professional technical ability, communication and coordination ability of practitioners, heavy workload and pressure, and pediatric nursing staff is in short supply, but professional development space is large. Among 216 interns, 182 (84.26\%) are willing to take up nursing work after graduation, of which 73 (33.8\%) are willing to take up pediatric nursing work. The research results indicate that nursing managers should take measures to improve the pediatric nursing practice environment, improve the salary and treatment of pediatric nursing staff, carry out pediatric communication skills training and situational exercises for nursing interns, improve their ability to deal with various communication scenarios and prepare for the transition of pediatric nursing practice for interns.
\end{abstract}

Keywords: Pediatrics, Nursing intern, Willingness to practice, Nursing cognition

\section{Introduction}

Pediatric nursing work is complex and difficult. The high requirements for nursing technology, the high expectations of parents, and the difficult communication between doctors and patients bring risks and challenges that are different from other clinical departments for daily nursing work. The shortage, turnover, and high turnover rate of pediatric nursing staff also bring great challenges to the development of pediatric nursing [1][2]. Nursing students are the main reserve force for nursing staff. Investigating the perception of nursing interns on pediatric nursing work and their willingness to engage in pediatric nursing work in the future will help to discover the problems and confusion they face in pediatric nursing practice. The teaching teachers provide the basis for teaching according to their aptitude and personalized guidance and finally realize the cultivation and reserve of pediatric nursing talents.

Article History:

Received (December 10, 2019), Review Result (January 18, 2020), Accepted (March 2, 2020) 


\section{Sample and Method}

\subsection{Sample of research}

216 nursing interns who graduated in 2018 and practiced in medical institutions were selected as the research sample. The inclusion criteria: graduated in 2018, have completed the school's professional theoretical courses; have internship experience in 2 or more departments of hospitals above level two; Internship time in pediatrics 2 weeks and above.

Exclusion criteria: The questionnaire did not provide the name of the internship hospital or the duration of the pediatric internship; except for pediatrics, who did not participate in the rotation of nursing practice in other departments; the pediatric internship time was less than 1 week.

\subsection{Survey Method}

\subsubsection{Survey tools}

The researchers designed and compiled the questionnaire of "Nursing Intern Pediatric Cognition and Practical Willingness Survey" based on consulting relevant literature and nursing textbooks, and revised it through repeated certification and preliminary experiments of nursing education experts.

The questionnaire consists of three parts:

(1) Basic information

The basic information includes gender, education, grade of internship hospital, pediatric internship time, and internship department other than pediatrics.

(2) Pediatric nursing cognitive questionnaire

It is composed of two parts: pediatric nursing content recognition and pediatric nursing work Feature recognition. Pediatric nursing content recognition includes the selection of pediatric nursing coverage content (multiple choice questions), pediatric nurse role ranking, pediatric nursing skill requirement ranking, and priority ranking of pediatric nursing principles.

The Features of pediatric nursing work include workload, work pressure, noisy workplace, level of violence, medical exposure risk, doctor-patient disputes, technical requirements, communication and coordination ability, staff shortage, salary and treatment, career achievement, and career future.

The development space has 12 dimensions, adopting the Liter 5-level scoring method, the lowest degree is 1 point, and the highest degree is 5 points.

(3) Questionnaire of pediatric nursing willingness to practice

Investigate whether interns are willing to work in nursing after graduation and whether they are willing to work in pediatric nursing.

\subsubsection{Data Collection}

Use the questionnaire star to create an electronic version of the questionnaire, and train the nursing teachers and interns of the researcher s unit to conduct initial training on the purpose and filling method of the questionnaire. The questionnaire method was snowball sampling among the interns. 
Questionnaire quality control method: note the questionnaire response notes, and the respondents fill in the questionnaire anonymously to ensure that the information is true and effective. Different medical institutions designate a survey team leader to be responsible for forwarding, filling out, and quality control of the questionnaire. Each questionnaire entry is set as a mandatory option, and there are missing items that cannot be submitted.

A total of 243 questionnaires were distributed and 243 questionnaires were recovered, including 216 valid questionnaires.

\subsection{Statistical processing}

The questionnaire software exports Excel data, establishes the original database, conducts quality check and missing value analysis, data analysis, and chart drawing adopt SPSS18.0 statistical software, continuous variables are described in the form of mean \pm standard deviation, and nominal variables are described in frequency and percentage.

\section{Results}

\subsection{Information on 216 nursing interns}

The information of 216 nursing inters is collected in [Table 1]. Females accounted for the vast majority of $90.28 \%$. For the current education information, college students accounted for the majority of $65.28 \%$ and undergraduates $32.87 \%$. Most internships have an internship period of 4 weeks $(60.65 \%)$ or more $(29.17 \%)$. The number of second-level and third-level hospitals is similar.

Table 1. Information of 216 nursing interns

\begin{tabular}{|c|c|c|c|}
\hline \multirow{2}{*}{ Gender } & Item & Sample number & Percentage (\%) \\
\cline { 2 - 4 } & Male & 21 & 9.72 \\
\hline \multirow{3}{*}{\begin{tabular}{c} 
Current Education \\
\cline { 2 - 4 }
\end{tabular}} & Female & 195 & 90.28 \\
\cline { 2 - 4 } & Technical secondary school & 71 & 32.87 \\
\hline \multirow{3}{*}{$\begin{array}{c}\text { Pediatric internship time } \\
\text { (weeks) }\end{array}$} & 2 & 4 & 65.28 \\
\cline { 2 - 4 } & 3 & 9 & 1.85 \\
\cline { 2 - 4 } & 4 & 13 & 4.17 \\
\cline { 2 - 4 } \begin{tabular}{c} 
Internship hospital level \\
\cline { 2 - 4 }
\end{tabular} & Second-level hospital & 131 & 6.02 \\
\hline \multirow{2}{*}{$\begin{array}{c}\text { Number of internships } \\
\text { in other departments }\end{array}$} & Third-level hospital & 63 & 29.17 \\
\cline { 2 - 4 } & $1-4$ & 68 & 31.48 \\
\cline { 2 - 4 } & $5-6$ & 74 & 68.52 \\
\hline
\end{tabular}

\subsection{Pediatric nursing cognition}

\subsubsection{Cognition of the content of pediatric nursing specialty}

In terms of pediatric nursing coverage, in addition to routine childhood disease protection, 95\% of interns think they should pay attention to children's growth and development, $96 \%$ of interns think they need to pay attention to child feeding, and $94 \%$ of interns believe that children 's physical and mental health should also be emphasized. 
In terms of the role of pediatric nurses, each intern ranks the role of pediatric nurses according to their importance. The number 1 indicates the first importance, and so on. Finally, the sequence number of each role is added together. The smaller the sum of the serial number values, the higher the importance of the role.

In the end, the pediatric intern thinks that the nursing role is ranked in order of care planner, professional caregiver, health educator, health coordinator, nursing researcher, children, and their spokesperson for the family.

The order of importance in terms of pediatric nursing skills is ideological and moral quality, scientific and cultural quality, professional skill quality, and physical and mental quality.

The order of importance in terms of pediatric nursing principles is to implement holistic physical and mental care, reduce trauma and pain, focus on children and families, abide by laws and ethics, and multidisciplinary collaborative care.

\subsubsection{Cognition of the Features of pediatric nursing work}

The survey confirmed that $\geq 60 \%$ of the interns agreed that pediatric nursing has the following Features: pediatric nursing requires high professional technical ability, communication and coordination ability of practitioners, heavy workload and pressure, and pediatric nursing staff is in short supply, but professional development space is large. Its cognitive scores are shown in [Table 2].

Table 2. 216 Interns' Cognitive Rating of Pediatric Nursing Work Features

\begin{tabular}{|c|c|c|c|}
\hline Feature & Score & Feature & Score \\
\hline Workload & $4.15 \pm 0.97$ & Skills requirement & $4.61 \pm 0.71$ \\
\hline Working pressure & $4.16 \pm 0.91$ & $\begin{array}{c}\text { Communication and } \\
\text { coordination skills }\end{array}$ & $4.50 \pm 0.68$ \\
\hline Noisy & $3.78 \pm 1.21$ & Staff shortage & $4.19 \pm 0.85$ \\
\hline Level of violence & $3.02 \pm 1.32$ & Remuneration & $2.91 \pm 1.73$ \\
\hline Medical exposure risk & $3.64 \pm 1.01$ & Career accomplishment & $3.68 \pm 1.02$ \\
\hline Doctor-patient dispute & $3.98 \pm 1.18$ & Future development space & $3.69 \pm 0.89$ \\
\hline
\end{tabular}

\subsection{Practice willingness of pediatric nursing}

Among 216 interns, $182(84.26 \%)$ are willing to take up nursing work after graduation, of which $73(33.8 \%)$ are willing to take up pediatric nursing work.

\section{Discussion}

The findings of this research show that most nursing interns agree that pediatric nursing work is rich in content, in addition to disease prevention, it also includes children's physical and mental health, growth and development, and feeding. Pediatric nurses play multiple roles in pediatric nursing, and the importance of various roles in the same work scene is not the same, and there is no unified standard answer. The results of nurse role ranking suggest that the importance of nursing planners is before professional caregivers, indicating that interns in this study can systematically view nursing work from the perspective of nursing management and health management, rather than pure nursing services, suggesting their nursing philosophy promotion. In the ranking of the importance of pediatric nursing skills requirements, ideological and moral qualities, and scientific and cultural qualities have 
priority over professional skill qualities, which reflects the intern's emphasis on their basic qualities and abilities, which is consistent with the basic requirements of employment units for professionals. Career interns with clear and mature workplace knowledge and strict requirements for their basic qualities. The surveyed nursing interns ranked pediatric holistic nursing as the top priority, indicating that in the current pediatric nursing education and working environment, the interns 'understanding is consistent with the health concept advocated by the World Health Organization, focusing on the overall physical and mental health status. Taken as a whole, nursing interns are relatively comprehensive in terms of nursing theory, which reflects the improvement of nursing education and the development of nursing concepts.

This study proves by way of quantitative cognition that nursing interns believe that the workload and pressure of pediatric nursing are relatively large. Engaging in pediatric nursing work means high technical difficulty and communication and coordination ability, pediatric nursing staff shortage, pediatric doctor-patient dispute prone, and large pediatric nursing career development space.

On the one hand, this is consistent with the results of previous surveys [3][4][5], and truly reflects the work of pediatric nurses. Health policymakers can use this as a reference to take effective measures to improve the pediatric nursing practice environment and attract more young people to the pediatric nursing team [6][7]; on the other hand, the above results also show that the pediatric nursing internship experience provides intern trainees with valuable training opportunities and helps them to fully understand the challenges they face in their future work, which is conducive to their knowledge and skills reserve for their nursing career after graduation [8].

This research survey found that only $33.8 \%$ of interns are willing to engage in pediatric nursing work after graduation. Combined with the general background of the "pain of pediatricians" prevalent in various medical institutions, this has put forward a huge challenge in pediatric nursing and child care.

This study also pointed out the direction for future nursing interns to teach. In the pediatric nursing environment, the children 's own communication and expression abilities are limited, emotions are unstable, and self-control ability is weak.

Parents' demand for children's physical and mental health services is higher, and the difficulty and complexity of pediatric care have increased significantly, which determines the long-term objective existence of the above status quo.

Pediatric nursing interns should be guided by the above-mentioned key factors, to solve the practical difficulties and problems faced by interns, and realize the student-centered nursing internship teaching model [9]. Teachers should carry out the training of communication skills between doctors and patients and scenario exercises, teach the means and methods of emotional management and emotional control, train interns the ability to confidently and calmly deal with various communication scenarios, and train interns to shield external interference.

Nursing interns should learn to establish a harmonious nurse-patient relationship with patients, help to form a virtuous circle, increase their sense of identity and job achievement in the nursing profession, and be more conducive to the development and growth of the pediatric nursing team [10]. 


\section{Conclusion}

In this study, we investigated the cognitive status of nursing interns in pediatric nursing through questionnaires. In terms of the connotation of pediatric nursing work, the theoretical cognition of the interns was relatively comprehensive; in terms of the characteristics of pediatric nursing work, the interns generally believed that the pediatric nursing workload and work pressure were large, and engaging in pediatric nursing work meant high technical difficulty and communication and coordination ability, the shortage of pediatric nursing staff, the easy occurrence of disputes between pediatric doctors and patients, and the great development space of pediatric nursing profession. From the perspective of the nursing talent reserve, nursing managers should take measures to improve the pediatric nursing practice environment, improve the salary and treatment of pediatric nursing staff, carry out pediatric communication skills training and situational exercises for nursing interns, improve their ability to deal with various communication scenarios and prepare for the transition of pediatric nursing practice for interns. The proportion of nursing interns willing to engage in pediatric nursing after graduation is not high, and the training of pediatric nursing talents has a long way to go.

\section{References}

[1] Li Li, "Impact of humanized management on pediatric nursing staff's turnover intention and job burnout," Nursing practice and research, vol.14, no.3, pp.92-93, (2017) DOI: 10.3969/j.issn.1672-9676.2017.03.039

[2] Zhang Yue-e, Peng Haiqi, "The application of previewing subject cases in the teaching of pediatrics nursing," Nursing practice and research, vol.15, no.3, pp.130-132, (2018) DOI: 10.3969/j.issn.1672-9676.2018.03.053

[3] Hu Jingxian, Huang Shuangli, Zhang Shumin, Zhang Qian, and Li Lihua, "Correlation between academic emotion, academic self-efficacy and professional identity of undergraduate nursing students," Journal of Mudanjiang Medical University, vol.39, no.5, pp.150-154, (2018)

[4] Gao Hong, Wu Baoyu Wu Chun, Wan Yanping, Feng Xiaoyan, and Peng Yan, "Analysis of the present situation and influencing factors of the professional willingness of pediatrics in nursing undergraduates," Journal of nursing administration, vol.18, no.11, pp.778-781, (2018) DOI: 10.3969/j.issn.1671315x.2018.11.005

[5] Kelsey Miller, Michael Monuteaux, Christopher Roussin, and Jochua Nagler, "Self-confidence in endotracheal intubation among pediatric interns: associations with gender, experience, and performance," Academic Pediatrics, vol.19, no.7, pp.822-827, (2019) DOI: 10.1016/j.acap.2019.06.013

[6] Wu Yufei, Mao Jingfu, and Wang Lin, "Study of nurses' evaluation of the magnetism nursing work environment and their quit intention in tertiary hospitals," Chinese Hospital Management, vol.38, no.5, pp.6466, (2018)

[7] Zhang Li and Jia Susun, "Problems and countermeasures of "Post-90s" pediatric interns" Journal of Traditional Chinese Medicine Management, vol.26, no.10, pp.162-164, (2018)

[8] David Strauser, Fong Chan, Elizabeth Fine, Kanako Iwanaga, Chelsea Greco and Cori Liptak, "Development of the perceived barriers scale: a new instrument identifying barriers to career development and employment for young adult survivors of pediatric CNS tumors," Journal of Neuro-Oncology, vol.13, pp.1-9, (2019) DOI: 10.1007/s11764-018-0722-8

[9] Liu Xia, "Periodic survey and analysis of nursing students' professional identity," Modern Diagnosis and Treatment, vol.28, no.6, pp.1175-1176, (2017)

[10] Su Qian, Wang Huizhen, Li Tao, and Wang Qiao, "Reform and application research of undergraduate nursing-patient communication teaching mode," Chongqing Medicine, vol.45, no.35, pp.5026-5027, (2016) DOI: $10.3969 /$ j.issn.1671-8348.2016.35.042 